\title{
Application of Dynamic Multi-stage Temporary Plugging Fracturing Technology in Directional Wells
}

\author{
Wang Jianhui ${ }^{1,2}$, Yin Guiqin ${ }^{1,2, ~ *, ~ D e n g ~ X i a n ' a n ~}{ }^{1,2}$, Li Xiaoyan ${ }^{1,2}$, Chen Qiang ${ }^{1,2}$, Xiang Kui ${ }^{1,2}$ \\ ${ }^{1}$ Oil \& Gas Technology Research Institute of Changqing Oilfield Co., Xi'an, China \\ ${ }^{2}$ Development of Low-Permeability Oil \& Gas Fields, National Engineering Laboratory for Exploration, Xi'an, China
}

\section{Email address:}

wjh7_cq@petrochina.com.cn(Wang Jianhui),yinguiqin_cq@petrochina.com.cn(Yin Guiqin), dxa1_cq@petrochina.com.cn(Deng Xian'an), 1xy100_cq@petrochina.com.cn (LiXiaoyan), chengq_cq@petrochina.com.cn(Chen Qiang), xky1_cq@petrochina.com.cn(Xiang Kui)

${ }^{*}$ Corresponding author

\section{To cite this article:}

Wang Jianhui, Yin Guiqin, Deng Xian'an, Li Xiaoyan, Chen Qiang, Xiang Kui. Application of Dynamic Multi-stage Temporary Plugging Fracturing Technology in Directional Wells. International Journal of Oil, Gas and Coal Engineering. Vol. 7, No. 4, 2019 , pp. 89-94. doi: 10.11648/j.ogce.20190704.11

Received: July 25, 2019; Accepted: August 26, 2019; Published: September 17, 2019

\begin{abstract}
At present, the mainstream technology for the stimulation of tight reservoir which exists a large number of interlayer is "perforation concentrated \& large-scale fracturing". But the fracture simulation and monitoring result indicates that the vertical zone of the reservoir actually isn't stimulated fully. To increase the degree of the reservoir stimulated, the stimulation mechanism of dynamic multistage temporary plugging fracturing, temporary plugging agent and technological parameter have been studied. By optimizing the combination of temporary plugging materials, dosage, construction parameters and adding speed, the temporary plugging material has stable performance, has played a good role in temporary plugging and boosting pressure in field tests. The critical technique which is characterized by "multi-cluster perforation, temporary plugging near wellbore and multistage fracturing" is developed, and the design concept of dynamic multi-stage temporary plugging fracturing pump injection is defined. The production of the test well is twice as the neighboring well, in addition, it can realize multistage fracturing in single operation to decrease the fracturing cost, which makes the technique a promising future.
\end{abstract}

Keywords: Tight Reservoir, Temporary Plugging, Multi-stage Fracturing, Perforation Concentrated, Appraisal Well

\section{Introduction}

At present, the reservoir taste of exploration and evaluation wells is getting worse year by year. Especially for thick tight heterogeneous layers, the idea of "perforation concentrated + general fracturing" is usually adopted in reservoir stimulation. Fracture simulation and monitoring results show that the whole oil layers can not be well fractured, and many poor layers are not fully utilized, resulting in no significant fracturing effect.

Dynamic multi-stage temporary plugging fracturing is based on general fracturing of large section oil layer, after multi-cluster perforation, dynamic selective plugging is carried out by degradable temporary plugging materials at each stage fracturing. After the completion of fracturing treatment in low stress area, the working fluid is forced to turn to high stress area, some reservoirs that have not been reformed or have not been fully reformed are stimulated completely, so the effectiveness of reservoir reform in oil layers are improved by multi-stage fracturing [1].

\section{The Stimulation Mechanism of Dynamic Multi-stage Temporary Plugging Fracturing}

This paper innovatively designs a dynamic multi-stage temporary plugging fracturing physical simulation test blocks. we "moves" the field fracturing process to the laboratory simulation, which realizes the simulation of fracture treatment of dynamic multi-stage temporary plugging. At the same time, we innovatively designed the physical simulation test blocks hole by using the prefabricated hole method, and performed the multi-cluster perforation fracturing physical simulation tests of casing completion. Through laboratory simulation, the 
initiation and propagation characteristics of multi-cluster perforation general fracturing and dynamic multi-stage temporary plugging fracturing about the thick tight reservoir are observed and analyzed, and the stimulation mechanism of dynamic multi-stage temporary plugging fracturing is demonstrated intuitively from the experimental point of view.

\subsection{The Analysis of Fracture Initiation Characteristics}

(1) Heterogeneous model and three-cluster perforation and general fracturing test

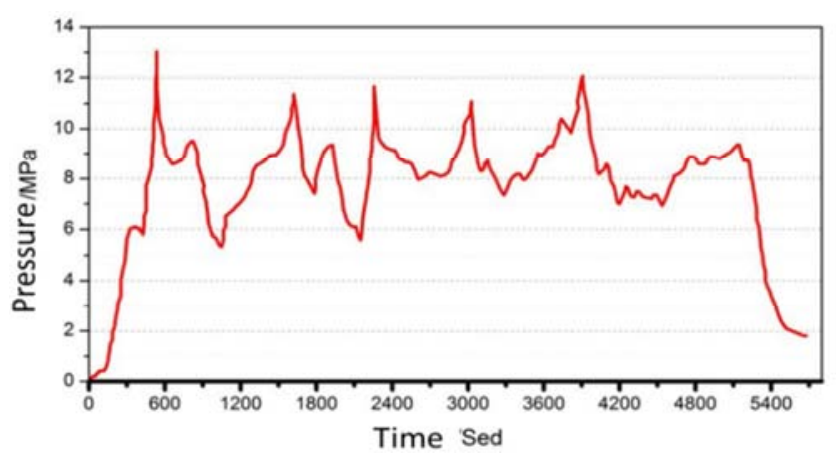

Figure 1. The experimental pump pressure curve of heterogeneous model + three cluster perforation + general fracturing test.

The pump pressure curve of the test are shown in figure 1. From the graph, it can be seen that there are many peaks of fracture break pressure, which is caused by the fracture initiation and extension from the multiple perforation sections. At 531 seconds, the first fracture initiation pressure is 13.0MPa, and then four peak pressures occur successively. The pressure fluctuation during the fracture extension process is large. On the one hand, the flow rate and pressure fluctuate due to the fracturing fluid flow at the multi-perforation sections, on the other hand, multi-fracture extension occurs simultaneously in heterogeneous test blocks, which makes the fracture extension pressure change.

(2) Heterogeneous model and three-cluster perforation and general fracturing test

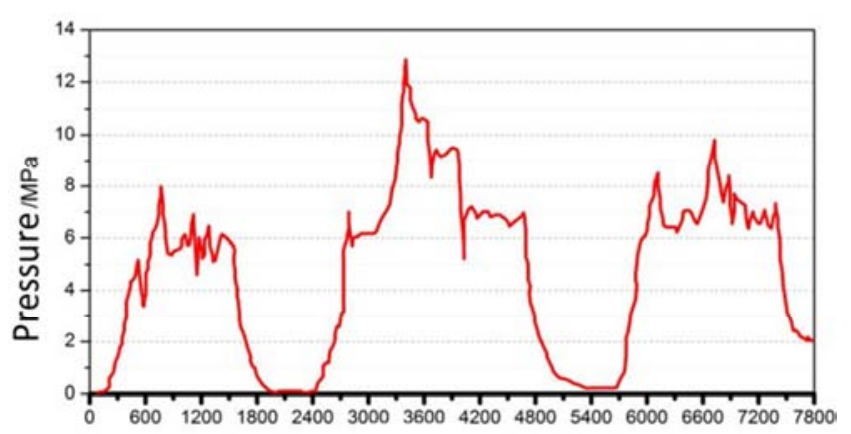

Time/Sed

Figure 2. The experimental pump pressure curve of heterogeneous model + three cluster perforation + temporary plugging + three- stage fracturing test.

The pump pressure curve of the test are shown in figure 2. The fracture initiated from the first stage perforation section at 771 seconds, and the break pressure is $8.0 \mathrm{MPa}$, the second stage fracture starts at $3402 \mathrm{~s}$, and the break pressure is $12.9 \mathrm{MPa}$, and the third stage fracture starts at $6725 \mathrm{~s}$, the break pressure reach at $9.7 \mathrm{MPa}$. The lower initiation pressure of the first and third stage fractures may be due to the close distance between the perforation location and the layer boundary and the weakening of the strength condition.

Through comparison, it is found that when multi-cluster perforation general fracturing in heterogeneous rock blocks, the fracture initiation and extension form is a single fracture surface, while when temporary plugging and graded fracturing, After the first-order fracture initiation and extension, the second-order and third-order fracture initiation and extension again, which further increases the complexity of the fracture.

\subsection{Fracture Propagation Law}

(1) Heterogeneous model and three-cluster perforation and general fracturing test

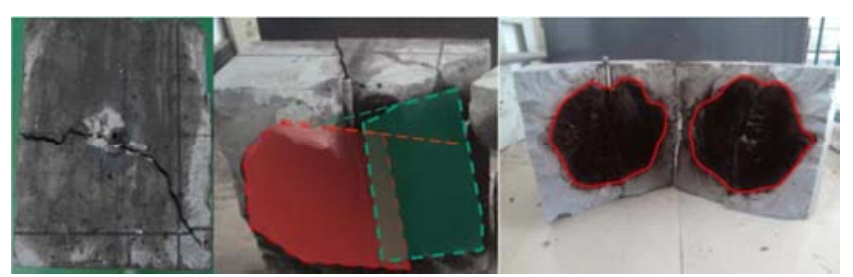

Figure 3. The fracture extension pattern of heterogeneous mode and three-cluster perforation and general fracturing test.

Fracture initiation and extension is recorded in Figure 3. After fracturing, the fracture path generally cracks along the direction of horizontal maximum principal stress. After fracturing from three perforation sections, the fractures are interconnected and the fracture basically presents symmetrical double-wing shape pattern, showing an elliptical profile as a whole. The fracture propagation area measured after fracturing accounted for $48.7 \%$ of the cross-sectional area of the test block.

(2) Heterogeneous model and three-cluster perforation and temporary plugging and three-stage fracturing test

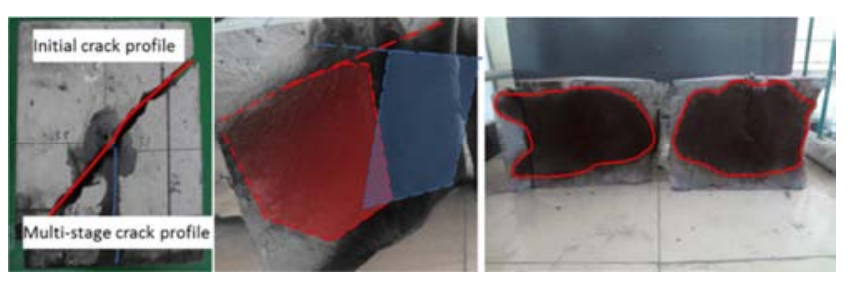

Figure 4. Fracture extension pattern of heterogeneous model + three-cluster perforation + temporary plugging + three-stage fracturing test.

As shown in Figure 4, we know that After fracturing from three perforation sections, the fractures are interconnected, and the fracture basically presents symmetrical double-wing shape pattern. The surface of fracture profile is rough and uneven, and fracture change way in its path at the the fracture tip. The main reason is that temporary plugging causes the fracture entrance compression and changes the local stress distribution. The fracture propagation area measured after fracturing accounted for $75.3 \%$ of the cross-sectional area of 
the test block. Its effect has obvious advantages over general fracturing under the same injection scale, and the expansion area of the fracture is greatly increased.

\section{Research on Dynamic Multi-stage Temporary Plugging Fracturing Technology}

Dynamic multi-stage temporary plugging fracturing technology is to improve bottom hole pressure through temporary plugging of open fractures, thus opening new reservoirs with higher stress. Temporary plugging uses different sizes of temporary plugging agent particles, degradable fibers and slugs mixed by them together. Large size particles are blocked at the entrance of hydraulic fractures. Small size particles are filled between large size particles to greatly reduce permeability and temporary plugging works. The addition of fibers ensures the integrity of the proportionally mixed particles from the ground to the bottom of the well and ensures the effectiveness of the temporary plugging, while not causing damage to reservoirs and fractures [2].

Dynamic multi-stage temporary plugging fracturing technology can improve wellbore contact area and reservoir reconstruction volume by sequentially reform different sections of perforation clusters or open hole wellbore. The technology can improve operation efficiency, productivity and ultimate recovery.

\subsection{Optimum Selection and Performance Evaluation of Temporary Plugging Materials}

Water-soluble temporary plugging agent is related to whether temporary plugging agent can effectively block up fractures and self-removal plugging can be achieved. The ideal temporary plugging agent has poor solubility at lower temperature, but better solubility at higher temperature. It can effectively temporarily block up fractures in the fracturing process. After completion of operation, the temperature near the wellbore rises and restores to formation temperature. At this time, temporary plugging agent dissolves rapidly and realizes self-removal plugging. In order to meet the requirement of that temporary plugging agent entering fractured fracture for temporary plugging inside fracture, water-soluble temporary plugging agent was selected for temporary plugging test evaluation [3-4].

(1) Compatibility evaluation of temporary plugging material and fracturing fluid

Temporary plugging particles become viscous and soft in linear gel and sink at the bottom of linear gel liquid. Fiber materials are well dispersed in linear gel liquid without agglomeration, precipitation or agglomeration.

(2) Solubility Evaluation of Temporary Plugging Agent and fiber under formation Conditions

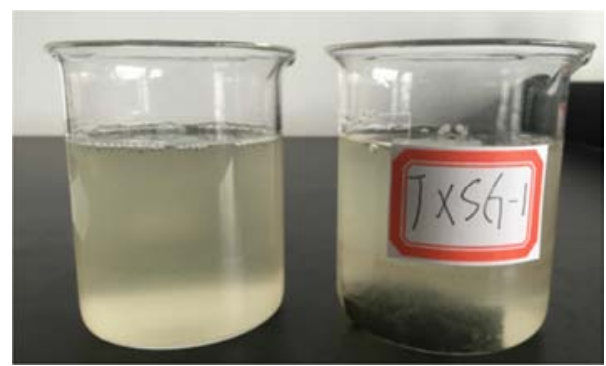

Figure 5. Dispersion of temporary plugging agent in linear gel.

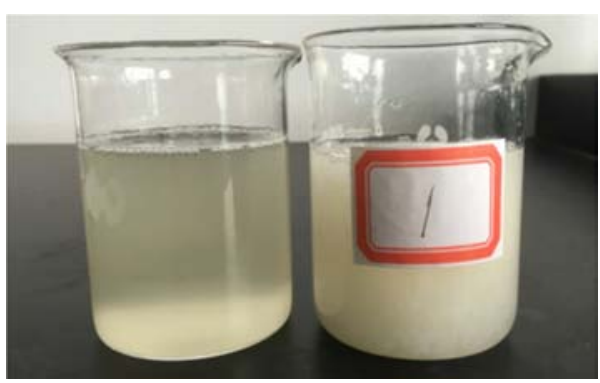

Figure 6. Dispersion of fiber material in linear gel.

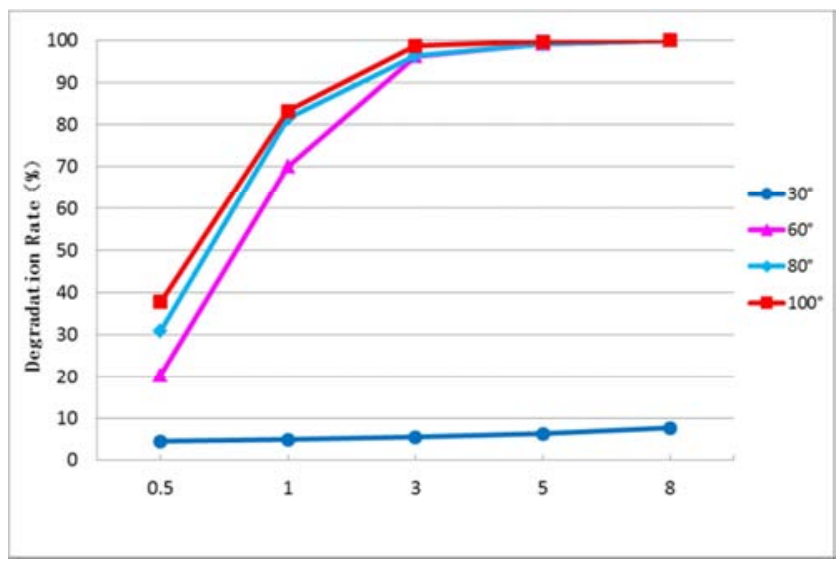

Figure 7. The degradation rate of temporary plugging agent at different temperature and time.

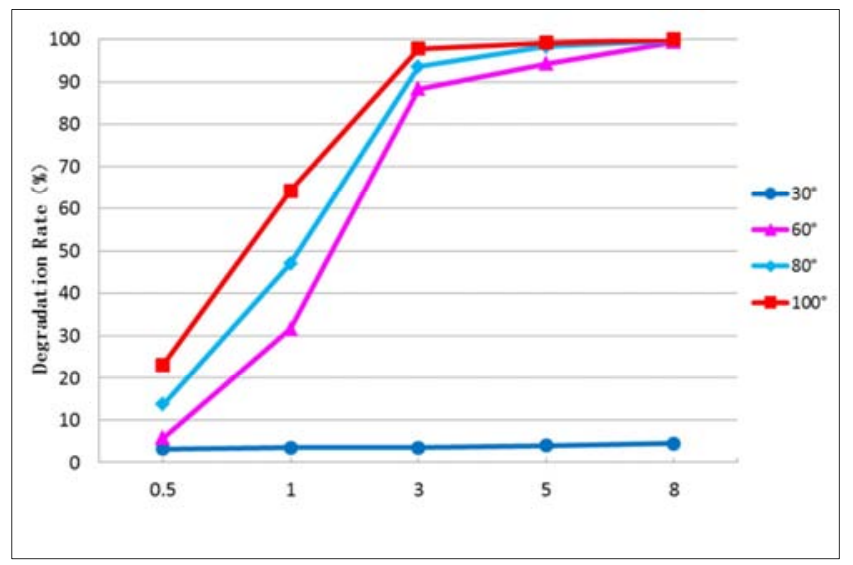

Figure 8. The degradation rate of fiber at different temperature and time.

As shown in figure 7 and figure 8 . The degradation rate of temporary plugging agent and fiber is about $1 \%-4 \%$ at $30^{\circ} \mathrm{C}$, which is difficult to degrade. When the temperature rises to $60^{\circ} \mathrm{C}, 80^{\circ} \mathrm{C}$ and $100^{\circ} \mathrm{C}$, the four temporary plugging agents 
degrade rapidly in the early stage, and the degradation rate reaches more than $90 \%$ in about 3 hours, and can be completely degraded between 5 hours to 8 hours. At the same time, the experimental results show that the higher the temperature, the faster the degradation of temporary plugging agent.

(3) Evaluation of pressure resistance of temporary plugging agent

Through core displacement test, a stirred and mixed temporary plugging agent with appropriate thickness is added between two natural cores. The core is placed in core holder, and the confining pressure of $3 \mathrm{MPa}$ is added. The core displacement device is used to displace the formation water forward at $0.1 \mathrm{ml} / \mathrm{min}$ flow rate. The pressure changes are observed at all times. The pressure value at the breakthrough time is recorded, and we continue to displace until the pressure is stable, then record pressure.

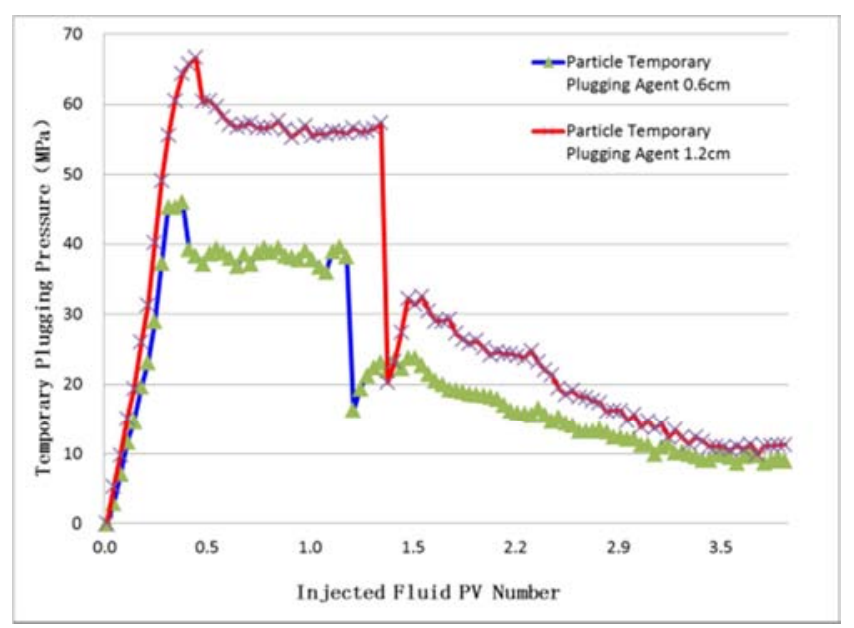

Figure 9. The relationship between PV number and temporary plugging pressure of injected fluid with different particle size temporary plugging agents.

As shown in figure 9 and figure 10, The curve of PV number of injected fluid with fiber and particle temporary plugging agent is similar to that of temporary plugging pressure. With the increase of the injection, fluid pressure significantly increased in the early period, reaching the highest press value, the fluid breaks through the temporary plugging slug, making the pressure reduction tends to balance gradually. $0.5 \mathrm{~cm}$ and $1.5 \mathrm{~cm}$ thickness fiber breakout pressures is $35.8 \mathrm{MPa}$ and $57.21 \mathrm{MPa}, 0.6 \mathrm{~cm}$ and $1.2 \mathrm{~cm}$ thickness particle temporary plugging agent is $45.01 \mathrm{MPa}$ and $65.07 \mathrm{MPa}$.
The effect of particle temporary plugging is slightly better than that of fiber temporary plugging. In the later period, With the extension of displacement time at $70^{\circ} \mathrm{C}$, the temporary plugging material gradually aged and lost its temporary plugging effect, which made the injection fluid pressure significantly decreased until the pressure is stable.

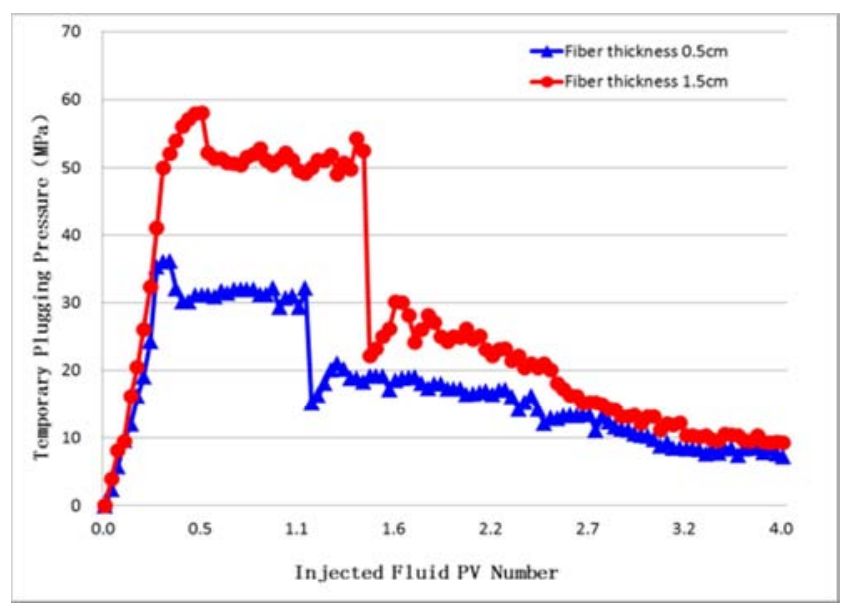

Figure 10. The relationship between $P V$ number and temporary plugging pressure of injected fluid with different thickness of fibers.

\subsection{Temporary Plugging Process Parameter Optimization}

In the early period, large-scale fracturing was used after cluster perforation with no obvious stimulation effect for the thick tight reservoir. To further improve the transformation effect, the key technology is to ensure the full utilization of reservoirs, and improve the dynamic multi-stage temporary plugging fracturing technology system by optimizing perforation treatments and fracturing parameters.

(1) Temporary plugging material combination optimization

From Table 1 can be seen, For water-soluble particles, water-soluble particles + fibers, water-soluble particles + fibers + proppants three combination modes, When the dosage of temporary plugging agent is fixed, the temporary plugging effect of water-soluble particles + fibers is the best, and the maximum temporary plugging fracturing pressure reaches 21.1 MPa. According to the experimental results, combined with construction experience, the temporary plugging agent slug is designed as: pre-fiber isolation fluid + water-soluble particles and fibers mixed temporary plugging fluid + post-fiber isolation fluid.

Table 1. Optimization results of dynamic multi-stage temporary plugging material combination type.

\begin{tabular}{lll}
\hline Temporary plugging material combination & dosage & maximum temporary plugging fracturing pressure \\
\hline water-soluble particles & $50 \mathrm{~g} / \mathrm{L}$ & $9.84 \mathrm{MPa}$ \\
water-soluble particles + fibers & $25 \mathrm{~g} / \mathrm{L}+25 \mathrm{~g} / \mathrm{L}$ & $21.1 \mathrm{MPa}$ \\
water-soluble particles + fibers + proppants & $20 \mathrm{~g} / \mathrm{L}+20 \mathrm{~g} / \mathrm{L}+10 \mathrm{~g} / \mathrm{L}$ & $19.8 \mathrm{MPa}$ \\
\hline
\end{tabular}

(2) Dosage of temporary plugging agent optimization

On the basis of laboratory experimental research of the temporary plugging agent performance evaluation, combined with the construction experience of temporary plugging fracturing field, the total amount of temporary plugging agent is designed according to the dosage of temporary plugging agent $1.5 \mathrm{~kg}$ per borehole.

$$
\mathrm{G}=1.5 \times \mathrm{L} \times \mathrm{D} \times \eta \times \mathrm{A}
$$


in which, G-per-stage temporary plugging fracturing plugging agent dosage;

L-perforation section length, m; D-hole density, hole/m; $\eta$-effective borehole;

A-temporary plugging agent dosage surplus coefficient [5].

\subsection{Construction Parameter Optimization}

(1) Temporary plugging agent adding method

Separation fluid and temporary plugging agent slug are designed from high-pressure pipeline to add, that is, after the first normal fracturing, stop pump, use special temporary plugging agent adding device to squeeze mixed temporary plugging material from high-pressure pipeline into wellbore.

(2) Temporary plugging agent adding speed

Combined with construction experience, in order to prevent the temporary plugging agent slug from being washed away in the wellbore migration process, the temporary plugging agent slug is designed to add with small flow which is $0.5-0.8 \mathrm{~m} 3 / \mathrm{min}$.

(3) Design of pump injection

Design ideas of dynamic multi-stage temporary plugging fracturing pump injection:

First stage fracturing $\rightarrow$ stop pump $\rightarrow$ temporary plugging isolating fluid $\rightarrow$ temporary plugging agent $\rightarrow$ temporary plugging isolating fluid $\rightarrow$ small flow displacement $\rightarrow$ second stage fracturing pre-fluid $\rightarrow$ stop pump $\rightarrow$ temporary plugging isolating fluid $\rightarrow$ temporary plugging agent $\rightarrow$ temporary plugging isolating fluid $\rightarrow$ small flow displacement......

\section{Field Test and Effect Evaluation}

Example of typical wells - dynamic multi-stage temporary plugging fracturing test wells:

As field fracturing construction curves show (A well, B well):

Through Field construction monitoring pressure changes reflect, in the four temporary plugging fracturing process, after temporary plugging agent reaches the perforation position, the bottom hole pressure increases by more than $10 \mathrm{MPa}$ on average, and decreases rapidly, with obvious fracturing breakout show. It shows that the following fracturing construction pressure and stop pump pressure at all levels are obviously increased, showing signs of new layer opening, indicating that new fractures open after temporary plugging of 4 stage in both two wells, to achieve the full use of the reservoir.

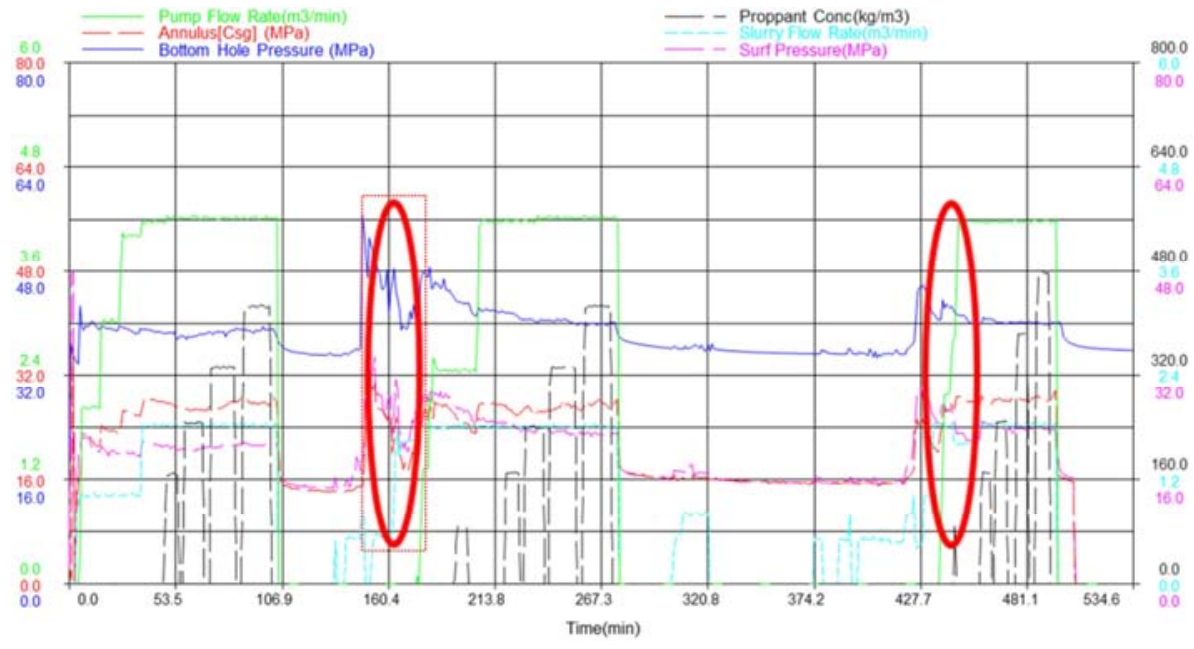

Figure 11. Field fracturing construction curve of Well A.

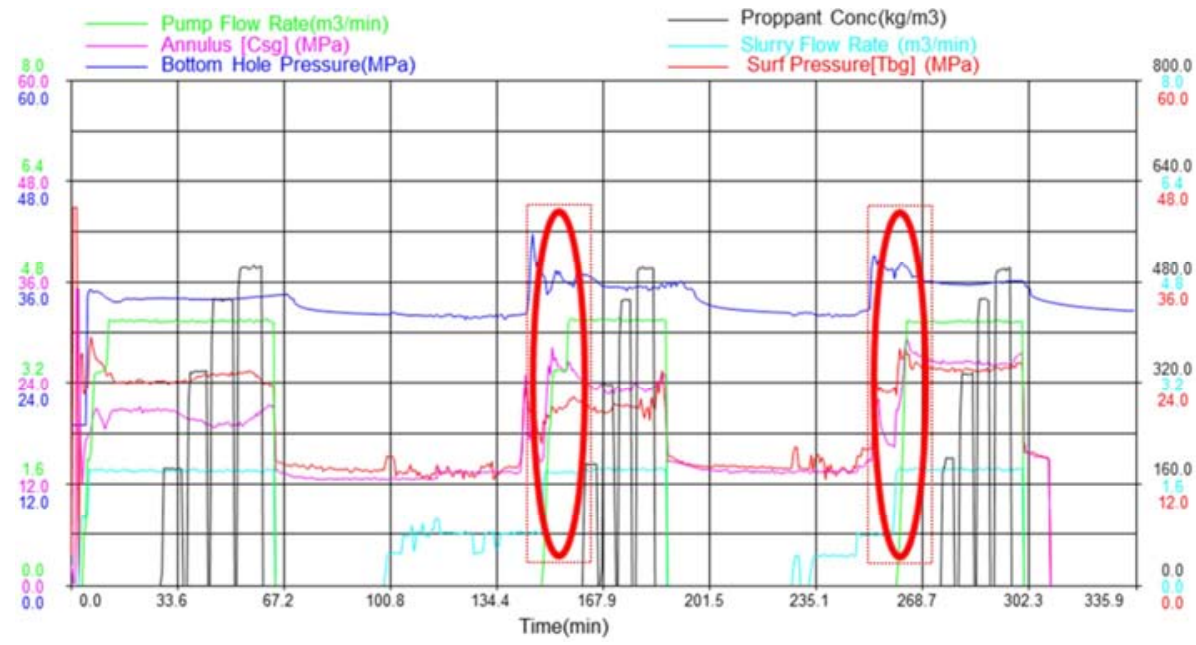

Figure 12. Field fracturing construction curve of Well B. 
2016-2017, 2 preferred test wells of 7-layer were selected in Huachi-Nanliang District. The average production per day of single test well was 41.1 tons. Four adjacent wells with similar reservoir conditions in the same area are selected as comparative wells, and the average production per day of four comparative wells is 17.62 tons. By comparison, it is found that the stimulation effect of the test wells is remarkable, and the stimulation range is more than $130 \%$. The test proves that dynamic multi-stage temporary plugging fracturing technology can further improve the full transformation of thick tight reservoirs, and the stimulation effect is remarkable.

\section{Conclusions and Understandings}

Through dynamic multi-stage temporary plugging fracturing physical simulation test, the initiation and propagation characteristics of multi-cluster perforation general fracturing and multi-cluster perforation dynamic multi-stage temporary plugging fracturing in thick tight layer are observed and analyzed, and the stimulation mechanism of dynamic multi-stage temporary plugging fracturing is demonstrated intuitively from the experimental point of view. Optimize stable fiber and temporary plugging agent. By optimizing the combination of temporary plugging materials, dosage, construction parameters and adding speed, a dynamic multi-stage temporary plugging fracturing technology for thick tight layers is formed, and the design concept of dynamic multi-stage temporary plugging fracturing pump injection is defined. Through indoor evaluation and field tests, it is found that temporary plugging material has good compatibility with fracturing fluid, good degradation performance and stable pressure performance under formation conditions, has played a good role in temporary plugging and boosting pressure, achieves the goal of fully utilizing reservoir by fracturing technology, and achieves good stimulation and injection effect.

\section{References}

[1] Xie Xinqiu, Zou Hongjiang, Wulong. Research and application of temporary plugging fracturing in low permeability oilfields [J]. Drilling and production technology, 2017, 40 (3): 65-67.

[2] Hu Jingtao. Research on technology of temporary plugging and volume transformation of tight reservoir [D]. Sichuan: Southwest Petroleum University, 2015.
[3] Dai Junhua, etc. Research and application of temporary plugging fracturing technology in Pingbei oilfield [J]. Drilling and production technology, 2006, 29 (6): 67-69.

[4] Wang Xiaoyu. Research and field application of water-soluble temporary plugging agent for fracturing $[\mathrm{J}]$. Petrochemical application, 2015, 34 (6): 92-94.

[5] Su Liangyin, Pang Peng, Da Yinpeng. The dosage optimization of temporary plugging repetitive fracturing agent and field test in low permeability oilfield [J]. Fault block oil and gas field, 2014, 21 (1): 114-117.

[6] Luo Hongyu, Fulton D D. Degradable Diverting Agents andAssociated Methods [P]. US: 8109335, 2012-02-07.

[7] Surjaatmadja J B, Todd B L. Fracturing Fluids ComprisingDegradable Diverting Agents and Methods of Use in Subter-ranean Formations [P]. US: 7506689, 2009-05-24.

[8] Warpinski N R, Teufel L W. Influence of geologic discontinuities on hydraulic fracture propagation [J]. Journal ofPetroleum Technology, 1987, 39 (2): 209-220.

[9] Blanton T L. Propagation of hydraulically and dynamicallyinduced fractures in naturally fractured reservoirs [R]. Louisville: SPE 15261, 1986: 1-15.

[10] Wang Zaiyu, Wei Xing, Feng Tian. Temporary Blocking Fracture Turning Research in Ansai Oilfield [J]. Liaoning Chemical Industry, 2014 (05): 587-588.

[11] Wang Ninfeng, Fu Zhenyin, Ren Yanpeng, et al. Application of multi-fracture fracturing technology in ultra-low permeability reservoirs [J]. Drilling and production technology, 2010, 33 (S1): 41-44.

[12] Yin Xiaohong, Zhou Zongqiang, Li Xianwen, et al. A temporary plugging agent for horizontal well fracturing and its preparation method [P]. China Patent: 2010134590.9, 2011-09-28.

[13] Zhou Fayuan, Pu Wanfen, Liu Chunzhi, et al. Developmentof temporary plugging agent for turning repeated fracturing. [J] Drilling and production technology, 2010, 33 (5): 111-113.

[14] Jiang Biwu, Mu Lijun. Performance of wax ball temporary plugging agent in repeated fracturing in low permeability oilfields [J]. Drilling and production technology, 2006, 29 (6): 114-116.

[15] Zang Haihong. Water-soluble fracturing acidizing temporary plugging agent [P]. Chinese patent: 200410009408.1, 2005-03-23.

[16] Zhang Jun, Wang Jianjun, Wen Yinwu, et al. Temporary Fracture Blocking Agent for Oil and Gas Reservoirs [P]. China Patent: 200710049168.1, 2008-11-26. 